\title{
Revision of the high-degree Gauss coefficients in the IGRF 1945-1955 models by using natural orthogonal component analysis
}

\author{
Wen-Yao Xu \\ Institute of Geology and Geophysics, Chinese Academy of Sciences, Beijing, 100101, China
}

(Received October 24, 2001; Revised May 10, 2002; Accepted June 11, 2002)

\begin{abstract}
The high-degree Gauss coefficients $(n \geq 7)$ in the IGRF models for 1945-1955 exhibit some unusual and unreliable behaviors in comparison with the models for other epochs. In this paper the method of Natural Orthogonal Components (NOC) is used to revise these coefficients. The obtained result shows that the main geomagnetic field can be approximated very well by the first a few NOC eigen modes. The high-degree Gauss coefficients $(n \geq 7)$ are deduced for each of IGRF models using the first 6 eigen modes and low-degree Gauss coefficients $(n \leq 6)$. The deduced high-degree coefficients are in very good agreement with the original ones for all models except those for 1945, 1950 and 1955. In comparison with the unusual behaviors in the IGRF19451955 models, the deduced high-degree coefficients for these models exhibit fairly smooth time-variations. Besides, the magnetic energy and magnetic flux calculated by the revised Gauss coefficients show more continuous secular variations and consistent characteristics.
\end{abstract}

\section{Introduction}

Since the adoption of the first International Geomagnetic Reference Field (IGRF) model, designated IGRF 1965, by IAGA in 1968 (IAGA Commission 2 Working Group 4, 1969; Zmuda, 1971), the IGRF series have been continuously extended forward to 2000 and backward to 1900 . These models have been widely used to study secular variations of the Earth's main magnetic field, dynamics in the Earth's core and space environment.

The time variations of the main magnetic field are believed to be continuous and fairly smooth. However, Barton (1997) noted some unusual variations in the high-degree Gauss coefficients (mainly for $n=7,8$ ) in the IGRF 1945-1955. $\mathrm{Xu}$ (2000) tested all Gauss coefficients up to $n=10$, and indicated that these unusual behaviors would lead to unreliable jumps in the magnetic energy and magnetic flux for 1945-1955. Lowes (private communication) attributed these unusual variations to that each of these models is based on an independent data set. These coefficients need revision to avoid misinterpretation of the secular variations.

A conventional approach to dealing with the problems is to re-examine the original data, which were used to create the models, and to re-calculate Gauss coefficients. Langel and Estes (1987) and Langel et al. (1988) collected all data available to them. By carefully considering the source and location of the survey data, local biases for the fixed observatories and the secular variation (SV) of the field, they established the models for 1945, 1950, 1955 and 1960, which were adopted as DGRF 1945-1960. We cannot expect better results than theirs, if the same procedure is repeated.

Another way is to fit the existing Gauss coefficients by, for

Copy right(C) The Society of Geomagnetism and Earth, Planetary and Space Sciences (SGEPSS); The Seismological Society of Japan; The Volcanological Society of Japan; The Geodetic Society of Japan; The Japanese Society for Planetary Sciences. instance, spline function (Sabaka et al., 1997), or to construct time-spatial models (Golovkov et al., 2000) for obtaining more or less smooth secular variations.

In this paper a new approach is pursued to improve highdegree Gauss coefficients of the IGRF models. Since the secular variations of the main field are believed to be continuous and fairly smooth, and its magnitude is much less than the main field itself, the main field has a relatively stable spatial structure. In this case, Method of Natural Orthogonal Components (NOC) can be applied to extract principle compositions of the main field from the existing series of the IGRF models (Leove, 1963; Kendall and Stuart, 1976; Frynberg, 1975; Pushkov et al., 1976; Rotanova et al., 1982; Galovkov et al., 1978; Sun et al., 1998, 2000). The obtained principle compositions are then used to reconstruct field models. After comparing the reconstructed models with the original IGRF and confirming their agreement, one can deduce highdegree Gauss coefficients from low-degree coefficients for IGRF 1945, 1950 and 1955.

\section{NOC Analysis of IGRF Models}

\subsection{Method of NOC}

Method of Natural Orthogonal Components (NOC) is widely used to calculate the principle compositions in a series of data samples (e.g. Yamada, 2002). In this paper the original data samples are 21 IGRF models for 1900-2000 at 5-year interval. The Gauss coefficients $g_{n}^{m}$ and $h_{n}^{m}$ (120 for each model) are directly used as the elements of the data sample (IAGA Division 5 Working Group 8, 2000). Using Langel's development to the present case (Langel, 1987), we let $V_{i j}$ represent the Gauss coefficients at epoch $t_{i}$, $i=1,2, \ldots, I(I \leq 21)$, and $j=1,2, \ldots, J(J=120)$. Thus, the original data can be expressed by a rectangular matrix $\mathbf{V}$ with $I$ rows and $J$ columns. An expansion is sought in 

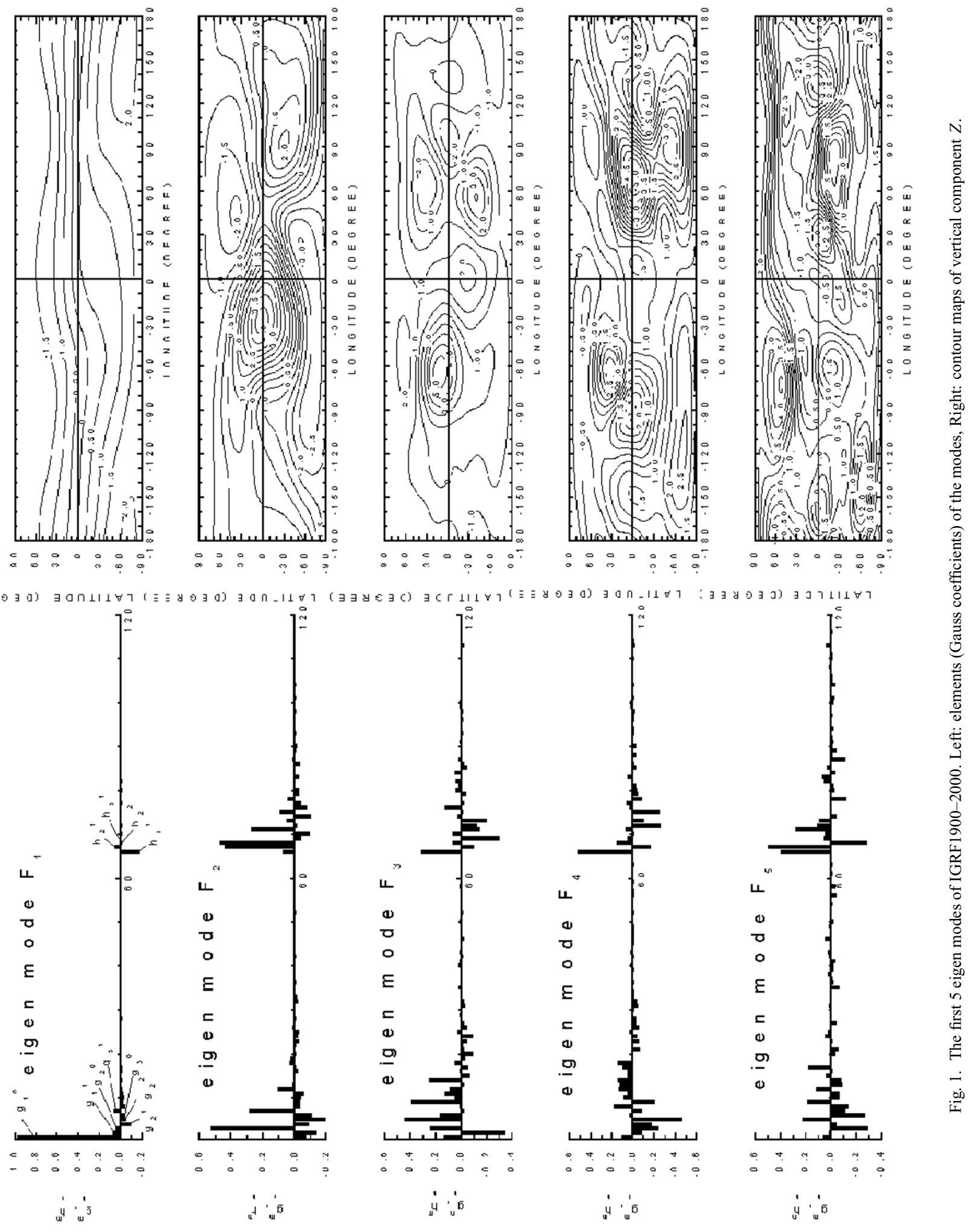
terms of functions $F_{k j}, j=1,2, \ldots, J, k=1,2, \ldots, K \leq$ $\min (I, J)$,

$$
V_{i j}=\sum_{k=1}^{k} A_{k i} F_{k j}+\delta_{i}^{j}(K)
$$

where $\delta_{i}^{j}(K)$ are the errors when we use truncated NOC series to fit the original Gauss coefficients. $F_{k j}$ are orthogonal over the data locations, i.e.

$$
\sum_{j=1}^{J} F_{k j} F_{l j}= \begin{cases}1 & k=l \\ 0 & k \neq l\end{cases}
$$

The amplitudes $A_{k i}$ in Eq. (1) represent the intensities of $F_{k j}$ and describe the contributions of $F_{k j}$ to the main field. They are functions of time with

$$
\sum_{i=1}^{I} A_{k i} A_{l i}= \begin{cases}\lambda_{k} & k=l \\ 0 & k \neq l\end{cases}
$$

To find $F_{k j}$ and $A_{k i}$, minimize

$$
\delta_{K} \equiv \sum_{i=1}^{I} \sum_{j=1}^{J}\left[\delta_{i}^{j}(K)\right]^{2}=\sum_{i=1}^{I} \sum_{j=1}^{J}\left[V_{i j}-\sum_{k=1}^{K} A_{k i} F_{k j}\right]^{2}
$$

with respect to $A_{k i}$ and $F_{k j}$. We obtain

where

$$
\mathbf{W F}_{k}=\lambda_{k} \mathbf{F}_{k}, \quad k=1,2, \ldots, K
$$

$$
\begin{aligned}
& \mathbf{F}_{k}=\left(F_{k 1}, F_{k 2}, \ldots, F_{k J}\right)^{T} \\
& \mathbf{W} \equiv \mathbf{V}^{T} \mathbf{V} .
\end{aligned}
$$

Equation (5) can be solved for eigen modes $\mathbf{F}_{k}$ and eigen values $\lambda_{k}$. Once $\mathbf{F}_{k}$ is found, the amplitudes $A_{k i}$ can be computed from the following equation

$$
\mathbf{A}_{k}=\mathbf{V F}_{k}
$$

Equation (1) indicates that each magnetic field model can be expressed by a summation of NOC terms, each of which has definite spatial structure $\mathbf{F}_{k}$ and varying intensity (or amplitude) $\mathbf{A}_{k}$.

\subsection{NOC analysis of all IGRF models for 1900-2000}

First of all, the method of NOC is used to all of 21 geomagnetic field models of the 8 th generation IGRF during 1900-2000 (IAGA Division 5 Working Group 8, 2000).

The eigen modes $\mathbf{F}_{k}$ are calculated and ordered according to decreasing eigen-value $\lambda_{k}$. In the left panel of Fig. 1 the elements (or Gauss coefficients) of the first 5 eigen modes $\mathbf{F}_{k}(k=1,2, \ldots, 5)$ are illustrated by columns, and are arranged according to the order of $g_{n}^{m}(n=1, \ldots, 10$, $m=0,1, \ldots, n), h_{n}^{m}(n=1, \ldots, 10, m=1,2, \ldots, n)$. In the right panel the corresponding contour maps of vertical component $Z$ are depicted for each $\mathbf{F}_{k}$. Obviously, the first eigen mode $\mathbf{F}_{1}$ represents the average pattern of all IGRF models, since the mean values of the Gauss coefficients for this period are not removed in the present calculation. Other modes show short wavelength structures, describing magnetic local anomalies with different spatial scales.
Figure 2 illustrates the time variations of amplitudes $\mathbf{A}_{k}$, and clearly shows the secular variations of the intensities of the modes $\mathbf{F}_{k}$. It is interesting to note that the variations of $\mathbf{A}_{k}$ exhibit different periodic characteristics: the magnitude of $\mathbf{A}_{1}$ decreases from $-32438 \mathrm{nT}$ in 1900 to $-30735 \mathrm{nT}$ in 2000 with a mean decay rate of $0.05 \%$ (or $17 \mathrm{nT} / \mathrm{a}$ ), same as the dipole moment decay rate, $\mathbf{A}_{2}$ varies from $1646 \mathrm{nT}$ to -1708 nT with a much greater rate of $2 \%$, while $\mathbf{A}_{3}, \mathbf{A}_{4}$ and $\mathbf{A}_{5}$ oscillate with periods $\sim 110, \sim 80, \sim 60$ years, respectively. These features imply some possible relationship between the time period and spatial wavelength in the main field.

A sharp decline of the magnitudes of $\mathbf{A}_{k}$ with $k$ is noted in Fig. 2, implying rapid convergency of the natural orthogonal series.

\subsection{NOC analysis of 18 IGRF models except for 1945 , 1950 and 1955}

In order to detect influences of the unreliable high-degree coefficients of IGRF 1945-1955 to eigen modes and eigen values, three IGRF models for 1945, 1950 and 1955 are removed from the data sample set, and NOC analysis is carried out again by using the rest of IGRF models.

The eigen values $\lambda_{k}$, eigen modes $\mathbf{F}_{k}$ and amplitudes $\mathbf{A}_{k}$ calculated from this reduced sample set are very similar to those from the complete sample set (see Figs. 1 and 2) for $k \leq 7$. This fact suggests that deleting 3 samples from all 21 samples has no essential influence to NOC analysis, implying stability of the principle eigen mode structures.

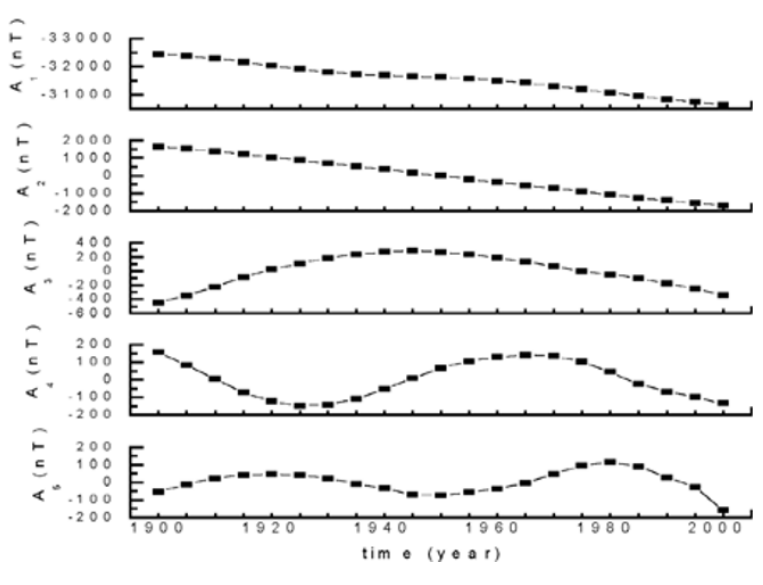

Fig. 2. Time variations of the intensities $\mathbf{A}_{k}$ for the first 5 eigen modes.

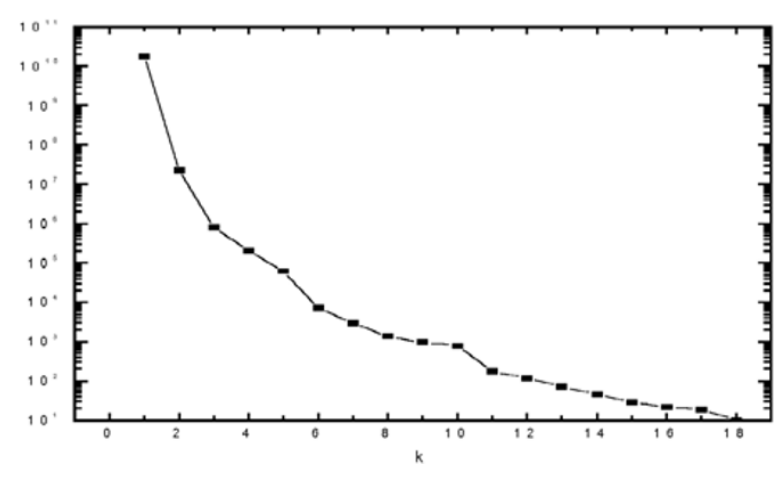

Fig. 3. Eigen values in NOC analysis of IGRF models. 

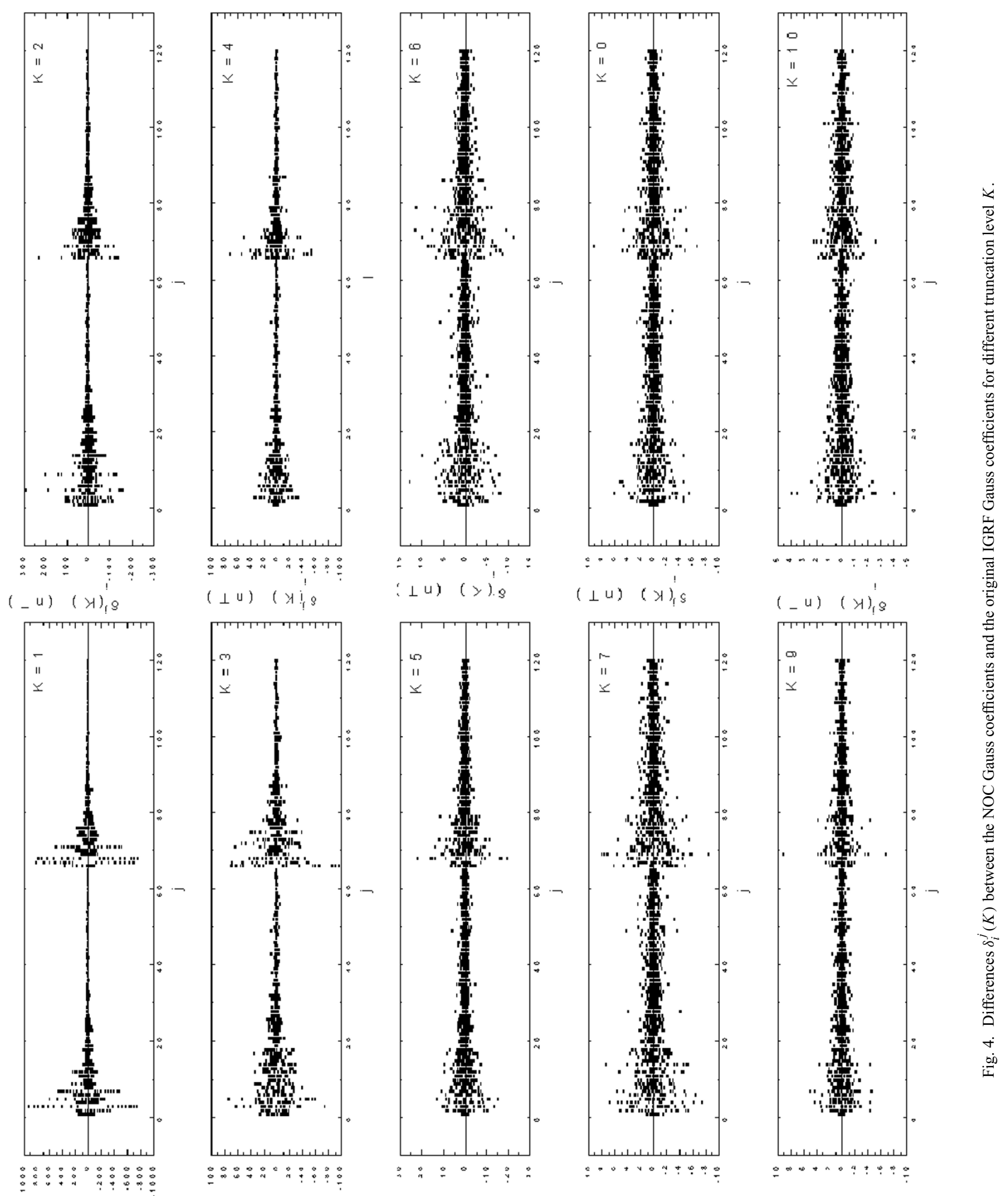
$(\perp u) \quad(x)_{1} \mathrm{~S}$

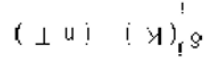

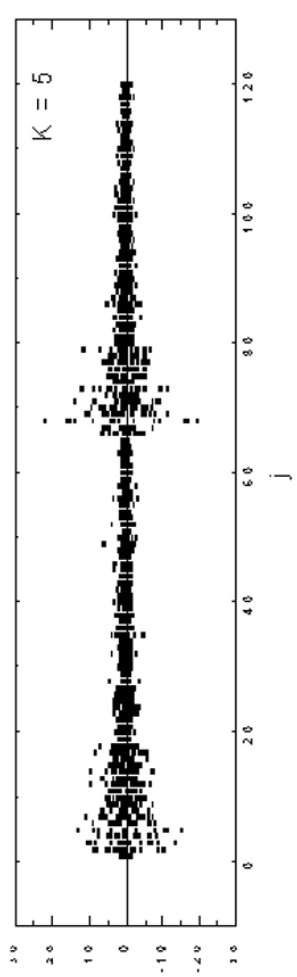

$(14) \quad(y), 8$

( 14$)(\forall) 3$ 
For a given truncation level $K$, one can construct NOC models of the main field from $\mathbf{F}_{k}$ and $\mathbf{A}_{k}$. Choice of $K$ is dependent upon the convergency of the series. As mentioned in Subsection 2.2, the amplitudes of eigen modes decline very rapidly with increasing $k$. This feature can be seen even more clearly in eigen values $\lambda_{k}$, as shown in Fig. 3 . The rapid decrease of the eigen values suggests that only the first a few terms in the NOC series (1) are important for the IGRF models, while other higher-degree NOC terms only have trivial contributions to the IGRF models.

In order to quantitatively assess the convergency of the NOC series, we calculate the Gauss coefficients of the NOC models for different truncation level $K$, and compare them with the original IGRF Gauss coefficients. In Fig. 4 the differences $\delta_{i}^{j}(K)$ of these two sets of Gauss coefficients for $K=1,2, \ldots, 10$ are shown, where the superscript $j$ of $\delta_{i}^{j}(K)$ are arranged according to the order of $g_{n}^{m}(n=1, \ldots, 10 m=0,1, \ldots, n), h_{n}^{m}(n=$ $1, \ldots, 10, m=1,2, \ldots, n)$, namely, $j=1,2,3, \ldots, 65$ for $g_{1}^{0}, g_{1}^{1}, g_{2}^{0}, \ldots, g_{10}^{10}$, and $j=66,67,68, \ldots, 120$ for $h_{1}^{1}, h_{2}^{1}, h_{2}^{2}, \ldots, h_{10}^{10}$. It is noted that $\delta_{i}^{j}(K)$ rapidly decrease with increasing $K$. For $K \geq 6$, most of $\delta_{i}^{j}(K)$ for lowdegree Gauss coefficients $(n \leq 5)$ are less than $5 \mathrm{nT}$, while most of $\delta_{i}^{j}(K)$ for high-degree coefficients $(n \geq 6)$ are less than $2 \mathrm{nT}$. In other words, the NOC series (1) converges very rapidly, and the IGRF models can be approximated very well by NOC models with a low truncation level.

\section{Calculation of High-Degree Gauss Coefficients from Low-Degree Ones}

In order to make distinction between the IGRF models and individual eigen models, we use notations $g_{n}^{m}\left(t_{i}\right)$ and $h_{n}^{m}\left(t_{i}\right)$ to represent IGRF Gauss coefficients, and use $g_{k}^{n m}$ and $h_{k}^{n m}$ to eigen modes. Equation (1) is rewritten as follows

$$
g_{n}^{m}\left(t_{i}\right)=\sum_{k=1}^{K} A_{k i} g_{k}^{n m}, \quad h_{n}^{m}\left(t_{i}\right)=\sum_{k=1}^{K} A_{k i} h_{k}^{n m} .
$$

The elements $g_{k}^{n m}$ and $h_{k}^{n m}$ of eigen modes have been obtained in Subsection 2.3. Using these eigen modes and the low-degree (for instance, $n \leq 6$ ) Gauss coefficients $g_{n}^{m}$ and $h_{n}^{m}$ of each IGRF model, one can solve Eq. (9) and obtain amplitudes $A_{k i}$. Equation (9) is usually an overdetermined family of equations. For instance, taking $K=6$ and $n \leq 6$, we have 6 unknowns $A_{k i}(k=1,2, \ldots, 6)$ and 48 equations.

Once $A_{k i}$ are obtained, all Gauss coefficients $g_{n}^{m}$ and $h_{n}^{m}$, including both low- and high-degree ones, can be deduced using Eq. (9). These deduced Gauss coefficients are called 'revised Gauss coefficients' or 'Gauss coefficients of NOC model'. The closeness of a NOC model to the original IGRF model is described by root-mean-square (rms) of the deviations of NOC Gauss coefficients from original IGRF coefficients. In Fig. 5 the rms deviations are shown for each model at different truncation levels $K$. It is noted that if $K \geq 6$, the rms deviations are close to the rounding error of the IGRF Gauss coefficients for all models except 1945, 1950 and 1955. This fact implies that the NOC models with $K \geq 6$ represent very well the original IGRF models. Considering the errors in IGRF models (Langel et al., 1988;

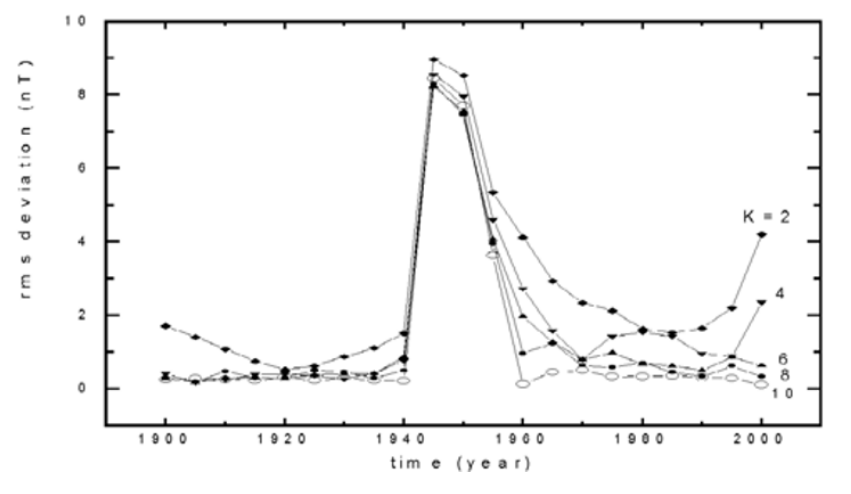

Fig. 5. Rms deviations of the revised Gauss coefficients for different truncation levels $K$.

Lowes, 2000), we set the truncation level $K=6$ in the following discussion. As for IGRF 1945, 1950 and 1955, the great deviations are attributed to unusual jumps of the highdegree Gauss coefficients of these models.

Figure 6 shows a comparison between the original (denoted by open circles) and revised (solid circles) Gauss coefficients $g_{10}^{m}$ and $h_{10}^{m}$ for $K=6$. The maximum revision (13 nT) occurs in $h_{10}^{3}$. It is noted that the unusual jumps in the original Gauss coefficients during 1945-1955 are removed in NOC models, while there are almost no revisions for other epochs.

In fact, ragged time-variations occur not only in the highdegree coefficients $(n \geq 7)$, but also in some lower-degree coefficients, for instance, $g_{4}^{3}, g_{5}^{0}, g_{5}^{2}, g_{6}^{0}, g_{6}^{4}, h_{5}^{5}, h_{6}^{3}$ (see figure 2 in Barton (1997), and figure 1 in $\mathrm{Xu}(2000)$ ). A complete comparison for all Gauss coefficients is made and shown partly in Fig. 7, in which the revised coefficients show fairly smooth variations.

The contour maps of the differences of $Z$ component between IGRF and NOC models are shown in Fig. 8 for 1945, 1950 and 1955, where the map for 1980 is also shown for comparison. The differences at most of points for 1980 are less than $10 \mathrm{nT}$, although the maximum difference is $40 \mathrm{nT}$, which is typical value for most of IGRF models. In contrast, the maximum differences for 1945, 1950 and 1955 are respectively 773, 532 and $345 \mathrm{nT}$. The great deviations for IGRF 1945, 1950 and 1955 are expected and attributed to unusual jumps of the high-degree Gauss coefficients of these models.

Rising the truncation level $K$ will lead to further decrease of rms deviation of the revised Gauss coefficients, although more ragged variations of the Gauss coefficients will occur.

\section{Test of the NOC Models}

In order to examine the NOC models, the magnetic energy beyond the core-mantle boundary is calculated by using the revised Gauss coefficients, and compared with those by the original IGRF models (Xu, 2000), as shown in Fig. 9. The unusual variations in the original 1945-1955 models are replaced by fairly smooth changes in the revised models. Similar comparisons are made for the magnetic multipole energy and for the magnetic flux at different depths. 


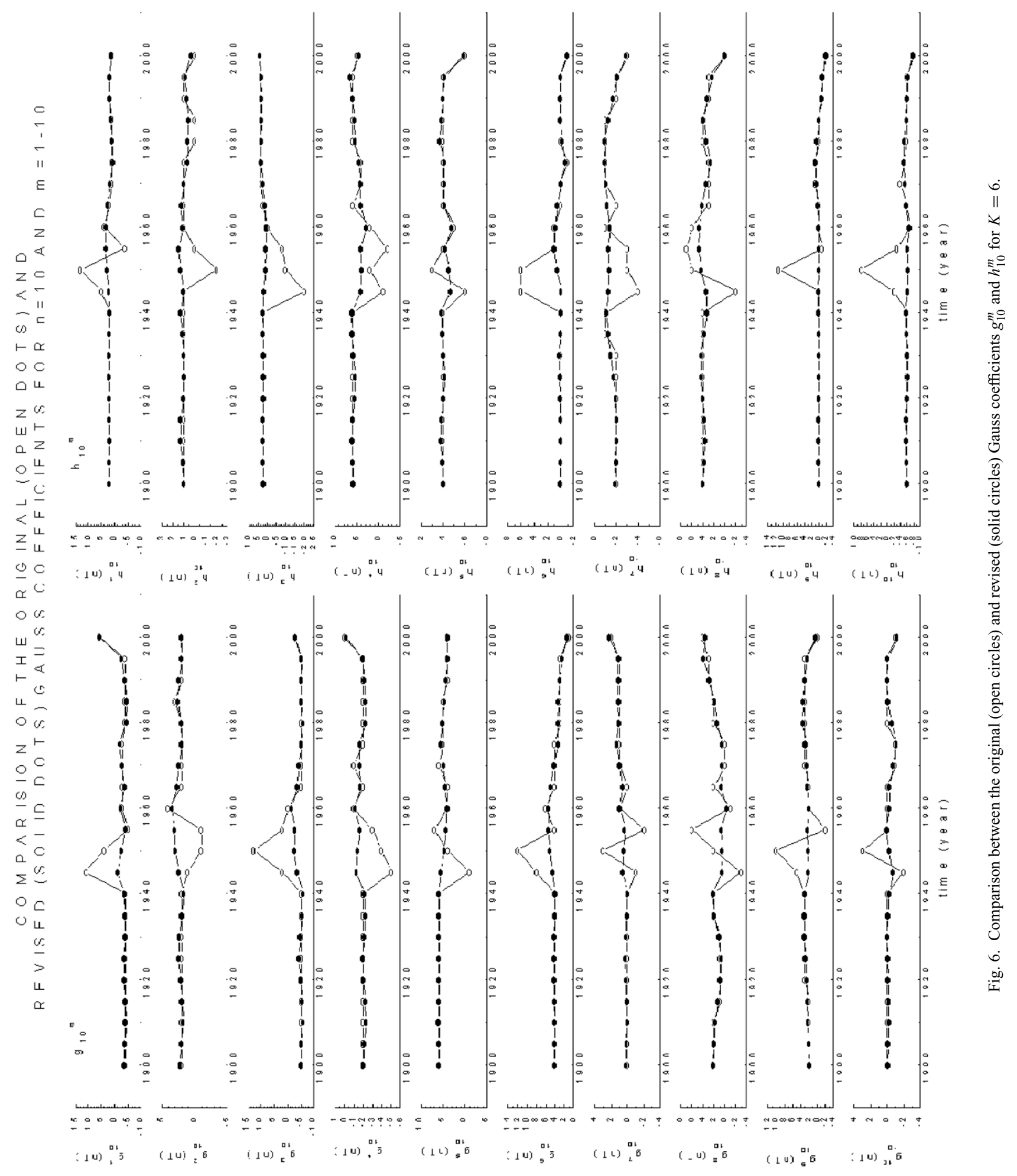




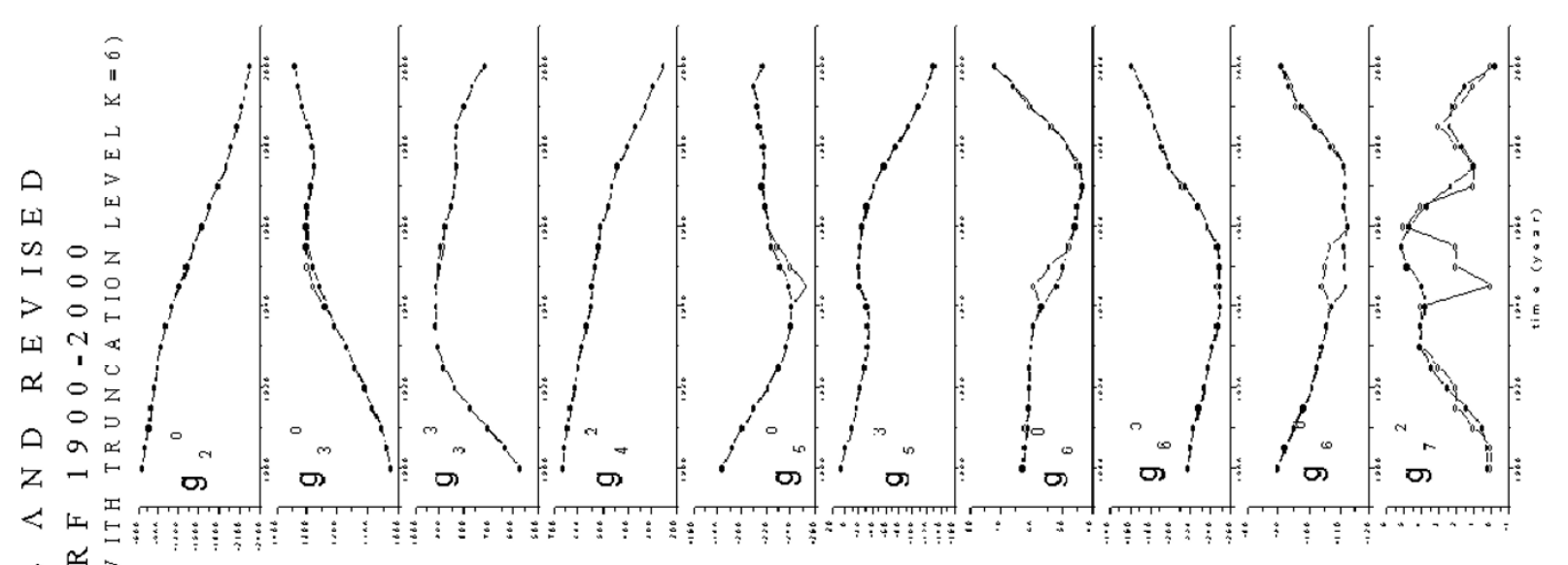

$\mapsto \infty$

$<\circlearrowright$

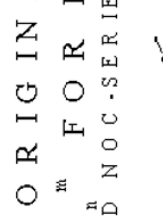

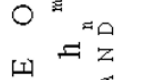

应

z of ${ }^{2}$

幽的苛

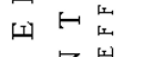

3 '
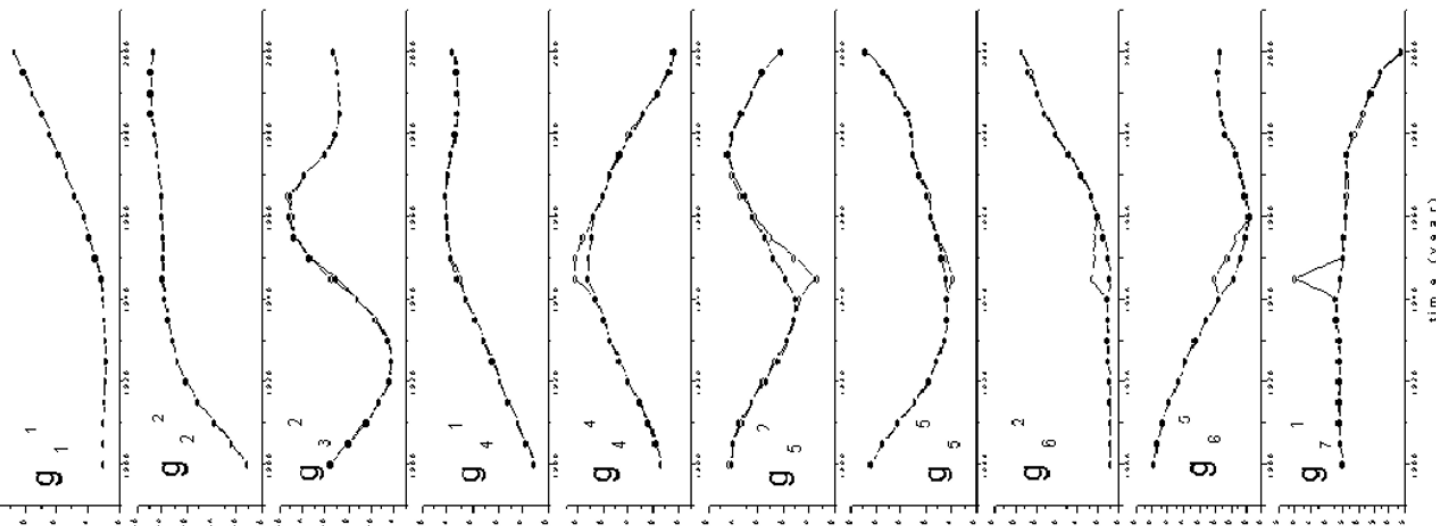

E

뇌 $U$ थ

の死方

乙近吕

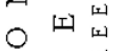

थ 0 皮

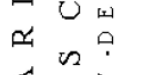

$<n$

ค.

$\sum \Psi_{0}^{\infty}$

○ 0

z
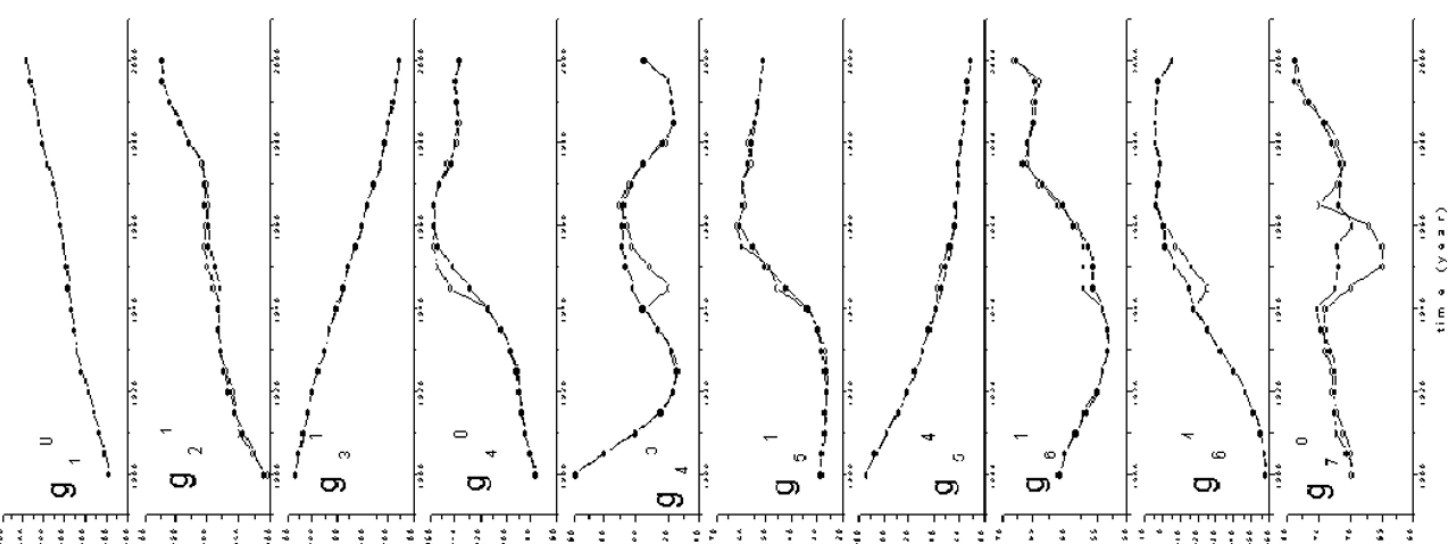

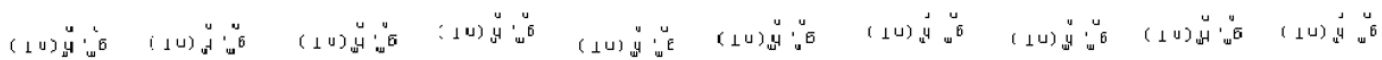



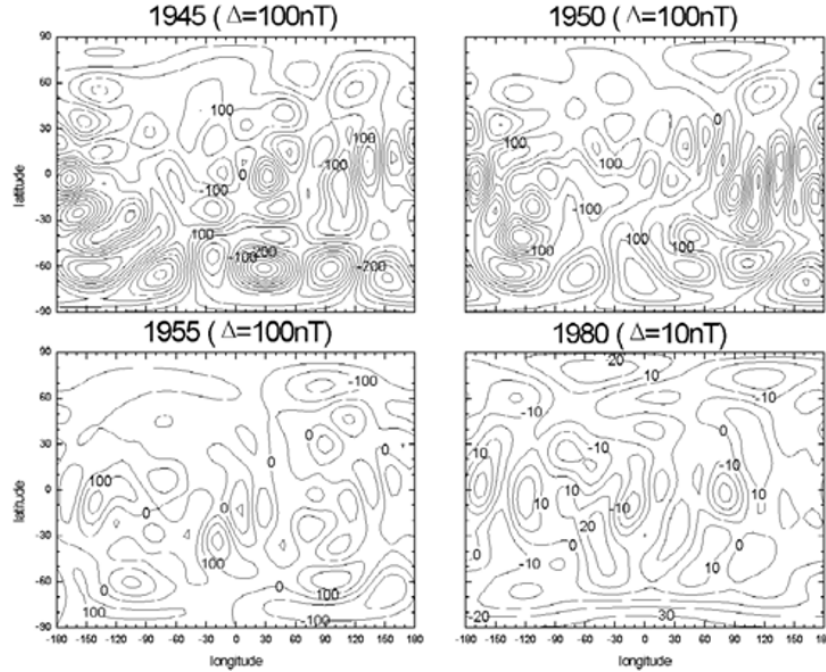

$1980(\Delta=10 \mathrm{nT})$

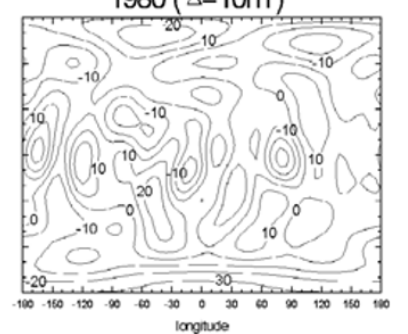

Fig. 8. The contour maps of the differences of $Z$ component between IGRF and NOC models for 1945, 1950, 1955 and 1980. The contour interval are $100,100,100$ and $10 \mathrm{nT}$, respectively.
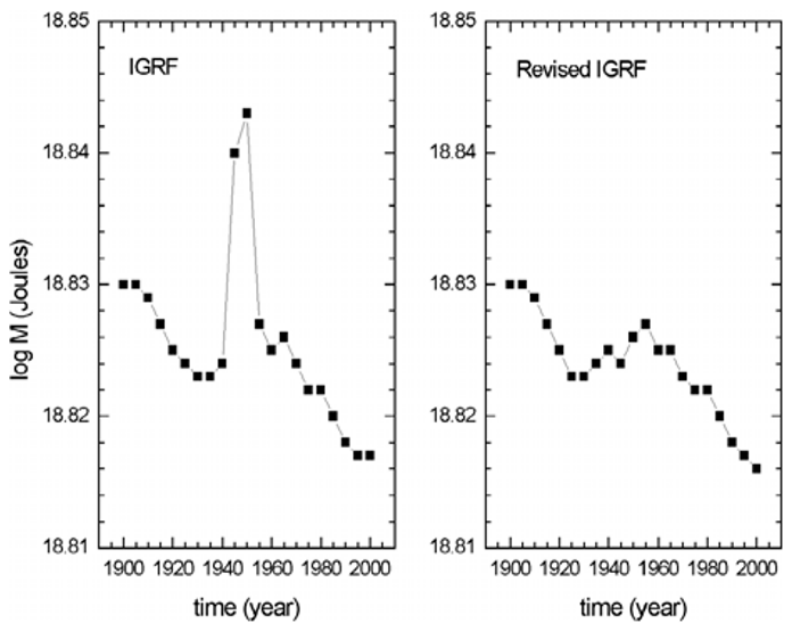

Fig. 9. Comparison of the magnetic energy beyond the core-mantle boundary calculated by using the original IGRF models (left) and the revised Gauss coefficients (right).

\section{Discussion and Summary}

The essence of NOC analysis is to reconstruct the main field model on the basis of the information in IGRF models, and to represent the field by the NOC series (1). It is shown that the NOC series converges very rapidly, and the main magnetic field can be approximated very well by the first a few lower-degree eigen modes, each of which includes all 120 Gauss coefficients. Consequently, the information of higher-degree Gauss coefficients is not lost. When we use lower-degree Gauss coefficients to determine the amplitudes (or intensities) of the eigen modes, we will obtain both lowdegree and high-degree Gauss coefficients.

Since the high-degree Gauss coefficients during 1945 1955 are questionable, the IGRF 1945-1955 are removed when we calculate the eigen modes. Fortunately, the deleting these 3 samples from all 21 samples has no essential influence to NOC analysis, as pointed out in Subsection 2.3. Only the reliable lower-degree Gauss coefficients of IGRF 19451955 are used to determine the amplitudes of eigen modes for these epochs.

The main results of this paper are summarized as follows:

1. NOC analysis of the IGRF 1900-2000 shows that the main geomagnetic field can be approximated very well by the first a few NOC eigen modes.

2. From the first 6 eigen modes and 48 low-degree Gauss coefficients $(n \leq 6)$ of the original IGRF models we deduced 72 high-degree Gauss coefficients $(n \geq 7)$. For all models except those of 1945, 1950 and 1955 the deduced highdegree coefficients coincide very well with the original ones, while for 1945, 1950 and 1955 the deduced high-degree coefficients show smooth time variations.

3. The magnetic energy and magnetic flux calculated by the revised Gauss coefficients show rather smooth variations in comparison with the unusual behaviors in original IGRF 1945-1955.

Acknowledgments. The author gratefully acknowledges W. Sun for supplying the IGRF data. This study is supported by the National Natural Science Foundation of China (49974014, 49734140) and The Ministry of Science and Technology of China (G20000784).

\section{References}

Barton, C. E., International geomagnetic field: the seventh generation, $J$. Geomag. Geoelectr., 49, 123, 1997

Frynberg, E. B., Separation of the geomagnetic field into a normal and anomalous part, Geomagn. Aeron., 15, 117, 1975.

Golovkov, V. P., N. E. Papitashvili, Y. S. Tyupkin, and E. P. Kharin, Separation of geomagnetic field variations on the quiet and disturbed components by the MNOC, Geomagn. Aeron., 18, 511, 1978.

Golovkov, V. P., T. N. Bondar, and I. A. Burdelnaya, Spatial-temporal modeling of the Geomagnetic field for 1980-2000 period and a candidate IGRF secular-variation model for 2000-2005, Earth Planets Space, 52 1125,2000

IAGA Commission 2 Working Group 4, International Geomagnetic Reference Field 1965.0, J. Geophys. Res., 74, 4407, 1969.

IAGA Division 5, Working Group 8, International Geomagnetic Reference Field 2000, Geophys. J. Int., 141, 259, 2000.

Kendall, M. G. and A. Stuart, The Advanced Theory of Statistics, vol. 3, chap. 35, Charles Griffin, London, 1976.

Langel, R. A., The Main Field, in Geomagnetism, Vol. 1, edited by J. A Jacobs, pp. 249-512, Academic Press, London, 1987.

Langel, R. A. and R. H. Estes, Derivation of proposed International Geomagnetic Reference Field models for 1945, 1950, 1955, and 1960, Phys. Earth Planet. Int., 48, 293, 1987.

Langel, R. A., D. R. Barraclough, D. J. Kerridge, V. P. Golovkov, T. J. Sabaka, and R. H. Estes, Definitive IGRF models for 1945, 1950, 1955 , and 1960, J. Geomag. Geoelectr., 40, 645, 1988.

Leove, M., Probability Theory, second edition, D Van Nosthand Company Inc., New York, 1963.

Lowes, F. J., An estimate of the errors of the IGRF/DGRF fields 1945-2000, Earth Planets Space, 52, 1207, 2000.

Pushkov, A. N., E. B. Frynberg, T. A. Chernova, and M. V. Fiskina, Analysis of the space-time structure of the main geomagnetic field by expansion into natural orthogonal components, Geomagn. Aeron., 16, 196, 1976.

Rotanova, N. M., N. E. Papitashvili, and A. N. Pushkov, Use of natural orthogonal components to distinguish and analyze the 60-yr geomagnetic field variations, Geomagn. Aeron., 22, 821, 1982.

Sabaka, T. J., R. A. Langel, R. T. Baldwin, and J. A. Conrad, The geomagnetic field 1900-1995, including the large-scale field from magnetospheric sources, and the NASA candidate models for the 1995 revision of the IGRF, J. Geomag. Geoelectr., 49, 157, 1997.

Sun, W., W.-Y. Xu, and S.-I. Akasofu, Mathematical separation of directly driven and unloading components in the ionospheric equivalent currents during substorms, J. Geophys. Res., 103, 11695, 1998.

Sun, W., W.-Y. Xu, and S.-I. Akasofu, An improved method to deduce the unloading component for magnetospheric substorms, J. Geophys. Res., 
105, 13131, 2000.

$\mathrm{Xu}$, W.-Y., Unusual behaviour of the IGRF during the 1945-1955 period, Earth Planets Space, 52, 1227, 2000.

Yamada, Y., 2-day, 3-day, and 5-6-day oscillations of the geomagnetic field detected by principal component analysis, Earth Planets Space, 54, 379, 2002 .
Zmuda, A. J., The International Geomagnetic Reference Field, 1965.0, in IAGA Bulletin 28, World Magnetic Survey, IUGG, Paris, 1971.

W.-Y. Xu (e-mail: wyxu@mail.o-geos.ac.cn) 